\title{
Reabilitação cognitiva em idosos institucionalizados: um estudo piloto*
}

\section{Cognitive rehabilitation on elderly institutionalized people: a pilot study}

\author{
Ana Paula Leal Loureiro ${ }^{1}$, Anne Abreu de Lima², Rita de Cássia \\ Gaspar da Silva ${ }^{3}$, Enise Cássia Abdo Najjar ${ }^{4}$
}

LOUREIRO, A. P. L., LIMA, A. A., SILVA, R. C. G, NAJJAR, E. C. A. Reabilitação cognitiva em idosos institucionalizados: um estudo piloto. Rev. Ter. Ocup. Univ. São Paulo, v. 22, n. 2, p. 136-144, maio/ago. 2011.

RESUMO: Este estudo descreve os efeitos da intervenção de terapia ocupacional no desempenho cognitivo, e analisa a influência deste desempenho na capacidade funcional de idosos institucionalizados. Trata-se de um estudo clínico randomizado, descritivo, longitudinal, realizado em uma instituição pública de longa permanência de idosos. Os 12 idosos que participaram do estudo foram divididos em dois grupos: experimental e controle. Para obtenção dos dados, foram utilizados: um questionário com dados sociodemográficos; o Mini-Exame do Estado Mental, o Teste de Fluência Verbal e o Teste do Relógio, para avaliação do desempenho cognitivo; e a Escala de Katz da Atividade de Vida Diária e o Questionário de Atividades Funcionais de Pfeffer, para avaliação da capacidade funcional. Os idosos do grupo experimental foram submetidos a uma intervenção com 23 sessões de reabilitação cognitiva. O grupo que recebeu a intervenção apresentou resultados estatisticamente significativos no desempenho cognitivo, mas não houve resultados estatisticamente significativos no desempenho da capacidade funcional quando comparados com o grupo controle. Conclui-se que a intervenção de terapia ocupacional teve efeitos significativos no desempenho cognitivo de idosos institucionalizados e que, provavelmente, o desempenho na capacidade funcional não foi tão significativo, por se tratar de idosos que, em sua maioria, apresentam limitação motora e dependência instalada.

DESCRITORES: Reabilitação; Terapia cognitiva/métodos; Idoso; Idoso fragilizado; Gestão em saúde; Saúde do idoso institucionalizado; Terapia Ocupacional.

\footnotetext{
* Este estudo compõe parte do trabalho de conclusão de curso de graduação em Terapia Ocupacional pela Universidade do Estado do Pará (UEPA).

1. Terapeuta Ocupacional graduada pela UEPA.

2. Terapeuta Ocupacional graduada pela UEPA.

3. Docente do Curso de Terapia Ocupacional da UEPA, doutoranda em Ciências do Desporto pela Universidade de Trás-os-Montes e Alto Douro (UTAD).

4. Docente do Curso de Terapia Ocupacional da UEPA, doutoranda em Teoria e Pesquisa do Comportamento pela Universidade Federal do Pará.

Endereço para correspondência: Tr. Barão do Triunfo, 3314/1302. Marco. 66093-050, Belém, PA. E-mail: enise@superig.com.br
} 


\section{INTRODUÇÃO}

$\mathrm{N}$ o Brasil, a demanda por instituições de longa permanência para idosos (ILPI) é crescente, à medida que a população brasileira envelhece. De um modo geral, a miséria e o abandono são os principais motivos para a internação dos idosos em asilos. Além disso, grande parte dos idosos é dependente, devido a problemas físicos e mentais (VERAS, 2003).

De acordo com a Gerência de Atenção a Pessoa Idosa, do Ministério de Desenvolvimento Social, no Brasil atualmente 24.964 idosos vivem em instituições nas redes conveniadas com o Ministério da Saúde (TIRADO; DRUMMOND, 2008).

Embora as portarias do Ministério da Saúde $\mathrm{n}^{\circ}$ 810/89 (BRASIL, 1989) e SAS n ${ }^{\circ} 73 / 01$ (BRASIL, 2001) tenham representado um grande avanço ao regulamentarem o funcionamento de ILPI no Brasil, o que se observa ainda é um panorama predominantemente desfavorável, freqüentemente associado ao tão criticado modelo asilar de atendimento ao idoso.

Estudos indicam que, raramente, encontram-se idosos que possuam intacta a autonomia e a independência em uma ILPI (SANTOS, 2006). A autonomia pode ser compreendida como a habilidade de tomar decisões pessoais sobre como viver o dia-a-dia, de acordo com suas regras e preferências, enquanto que a independência é a capacidade de concretizar as atividades de vida diária (AVDs) sem ou com pouca ajuda de outros (HARGREAVES, 2006).

Uma grande proporção de idosos asilados tem comprometimentos físicos e mentais (CHAIMOWICZ, 1997). Uma pesquisa com idosos em situação de institucionalização, realizada na cidade de Jequié, Bahia, apontou que $71,6 \%$ dos idosos apresentaram algum comprometimento funcional (REIS et al., 2008).

Estudos destacam que a realização de atividades minimiza os efeitos da institucionalização. O desenvolvimento de atividades de lazer em horários livres, em instituições, aumenta a auto-estima e a participação social dos idosos. Porém, a realização de atividades esporádicas e sem investigar as reais necessidades promove um benefício momentâneo e sem durabilidade para a saúde integral do idoso (JOÃO et al., 2005; SANTANA et al., 2008).

$\mathrm{O}$ equilíbrio entre a manutenção do desempenho cognitivo e da capacidade funcional garante ao idoso manter sua participação nas atividades cotidianas, preservando a qualidade de vida e aumentando o senso de bem-estar, através do desenvolvimento da competência e do controle de si mesmo e do seu ambiente. Estratégias com este objetivo são pertinentes, necessárias e urgentes, uma vez que as instituições asilares continuarão sendo importantes ambientes de moradia de idosos (CARDOSO; FREITAS; TIRADO, 2002; SOUSA; LOVISI, 2007).

Em idosos que vivem em ambientes institucionais, o ócio e a inatividade são comuns, predominando a desocupação e a manutenção da disfunção, além da diminuição da interação com o meio e a perda de contatos sociais. Este fato ocorre devido, principalmente, a dois fatores: (1) às incapacidades, uma vez que a mobilidade e a energia no idoso tendem a diminuir; e (2) ao isolamento, por ser um ambiente restrito (ARRIBADA, 2004).

Assegurar a manutenção dos idosos em ocupações, com uma abordagem estimulante e de qualidade, é de vital importância na intervenção, pois pode existir um elevado número de acontecimentos físicos, emocionais, sociais e ambientais afetando o sujeito ao mesmo tempo, dificultando a preservação de sua independencia (ARRIBADA, 2004).

Neste contexto, o terapeuta ocupacional desempenha um papel fundamental, que objetiva ajudar pessoas com deficiências cognitivas a estabelecer ou manter uma vida significativa e produtiva, dentro de seu ambiente social e cultural (AMERICAN OCCUPATIONAL THERAPY ASSOCIATION, 1999). Por meio do resgate e da estimulação do idoso nas atividades cognitivas, bem como a organização de seu cotidiano, o processo terapêutico ocupacional envolve quatro etapas: triagem e avaliação, criação e apresentação do plano de reabilitação, tratamento e, por fim, alta e acompanhamento (MELLO apud CAVALCANTI; GALVÃO, 2007).

No tratamento em reabilitação cognitiva usamse atividades que mantenham ativas a concentração, a seqüência de pensamento, a atenção e a capacidade de fazer escolhas do idoso. Pelas atividades, este é estimulado a utilizar suas capacidades remanescentes e mantê-las, convertendo-se em um trabalho de manutenção e prevenção (BARRETO; TIRADO apud FREITAS et al., 2002).

Várias técnicas para a atuação prática na terapia ocupacional são propostas, dentro da reabilitação cognitiva. Otero e Scheitler (2001) apresentam as técnicas e estratégias na reabilitação cognitiva, a partir de três níveis: (1) restauração: as funções cognitivas alteradas são estimuladas e melhoradas pela ação direta sobre elas; (2) compensação: assumindo que a função alterada não pode ser restaurada, tenta-se potencializar o emprego de diferentes mecanismos alternativos ou de habilidades preservadas; (3) substituição: ensino de diferentes estratégias que ajudem o sujeito a minimizar os problemas resultantes das disfunções cognitivas.

Destacamos neste estudo, as técnicas para o tratamento individual de pacientes empregadas pelo Setor 
LOUREIRO, A. P. L. et al. Reabilitação cognitiva em idosos. Rev. Ter. Ocup. Univ. São Paulo, v. 22, n. 2, p. 136-144, maio/ago. 2011.

de Neurologia Comportamental e Demências do Hospital Universitário Antonio Pedro (HUAP) da Universidade Federal Fluminense (UFF) e pelo Programa Interdisciplinar de Geriatria e Gerontologia (PIGG), da mesma universidade, por enfocarem o desempenho ocupacional, e por promoverem a interação entre o sujeito e o ambiente. Estas técnicas dividem-se em 5 tipos (CAMARA et al., 2009):

- Técnicas comportamentais: Considera que as respostas comportamentais e emocionais podem ser condicionadas a certos estímulos que são indutores de respostas específicas, envolvendo reforço positivo. Estimulam a mudança de comportamento indesejado do paciente, potencializando a participação nas atividades de vida diária e a socialização.

- Terapia de Orientação para a Realidade: Deve-se evocar o passado para significação do "eu", como forma de prazer e lembranças positivas. No presente, promover a independência, a orientação temporal e estimular a socialização. No futuro, despertar o interesse em novos projetos de vida. Sugere-se, em atividade, utilizar calendários, relógios, nomes e ambientes do cotidiano do paciente, reforçando repetições em busca do aprendizado e da satisfação do paciente.

- Estimulação Cognitiva: Com base na capacidade de adaptação e na plasticidade cerebral, deve-se estimular o que estiver conservado, compensar o que foi perdido e desenvolver potenciais remanescentes. As atividades propostas devem apresentar ludicidade, favorecer os atos da vida cotidiana, utilizando cores, dias da semana, mês e ano, hora, sabores.

- Terapia de Reminiscências: Permite a valorização do passado e do conhecimento do paciente, favorecendo as conversas, ordenando cronologicamente fatos importantes e lembranças significativas da vida. Utilizar canções antigas, cartas, fotos, discos, presentes, álbuns de família, filmes, jornais, músicas, proporcionando lembranças agradáveis.

- Técnicas de Validação: Objetiva oferecer suporte emocional e sentido às expressões dos pacientes. "Proporcionar alívio de desconfortos emocionais e de conflitos relacionais. Visa ao aumento da atenção, do autocontrole, da auto-estima e ao envolvimento do paciente no contexto social natural", de modo lúdico.

\section{OBJETIVO}

Este estudo se propõe a descrever os efeitos da intervenção de terapia ocupacional, utilizando a reabilitação cognitiva, no desempenho cognitivo; e observar a influência na capacidade funcional de idosos institucionalizados.

\section{MÉTODO}

Estudo clínico randomizado, descritivo, longitudinal, realizado em 2009, em uma instituição pública de longa permanência de idosos em Belém, no estado do Pará.

O estudo foi aprovado pelo Comitê de Ética em Pesquisa Envolvendo Seres Humanos da Universidade do Estado do Pará em 12/2008 sob o protocolo de no 176/08 e autorizado pela direção da instituição.

\section{Participantes}

Participaram da pesquisa 12 idosos com idade igual ou superior a 60 anos, regularmente internados na instituição, em situação de abrigamento permanente, após assinarem o Termo de Consentimento Livre e Esclarecido. Foram excluídos idosos acamados e com afecções neurológicas prévias em decorrência destas condições afetarem as respostas nos testes realizados.

\section{Instrumentos}

A coleta de dados foi realizada por meio de seis instrumentos, constituídos por: (1) um questionário para obtenção das variáveis sócio-demográficas (idade, sexo, tempo de institucionalização, motivo de institucionalização, recebimento de visitas, naturalidade, escolaridade e renda); (2) o Mini-Exame do Estado Mental (MEEM) para a avaliação da capacidade cognitiva geral; (3) o Teste de Fluência Verbal para avaliar a memória, a função executiva e a linguagem; (4) o Teste do Relógio que avalia diversas funções cognitivas, como memória e função executiva (APRAHAMIAN et al., 2009); (5) a Escala de Katz da Atividade de Vida Diária (KATZ et al., 1970), para avaliar a capacidade funcional em 7 funções (vestir-se, banhar-se, usar o banheiro, transferir-se, alimentar-se e controlar os esfíncteres); (6) o Questionário de Atividades Funcionais de Pfeffer, para avaliar a funcionalidade de idosos em desenvolver atividades instrumentais em casa e na comunidade (PFEFFER, 1982).

Os participantes foram distribuídos de forma aleatória em dois grupos: o grupo experimental formado por 6 idosos que receberam a intervenção, e o grupo controle, formado por 6 idosos que não receberam a intervenção. Em seguida, procedeu-se a aplicação dos instrumentos a todos os idosos, tendo sido reaplicados ao final da intervenção realizada.

O grupo experimental recebeu uma intervenção previamente planejada. O período da intervenção durou 16 
semanas, portanto 4 meses, sendo realizadas 23 sessões de reabilitação cognitiva, durante 2 dias por semana, com tempo médio de 45 minutos de duração. Das sessões realizadas, 17 se caracterizaram por atendimentos individuais e 6 por atendimentos grupais.

Utilizou-se nas intervenções os três níveis de estratégias da reabilitação cognitiva: a restauração, a compensação e a substituição. Além disso, foram empregadas cinco diferentes técnicas, distribuídas na seguinte forma: 6 sessões em que se utilizou a técnica de terapia de orientação para a realidade; 5 sessões em que se aplicou a técnica de terapia de reminiscências; 6 sessões com técnica de estimulação cognitiva; 4 sessões com técnicas de validação; 2 sessões utilizando as técnicas comportamentais.

Após a execução completa da intervenção com o grupo experimental, por questões éticas, foi iniciado o atendimento do grupo controle, empregando-se o mesmo procedimento realizado com o grupo experimental.

A análise estatística teve como objetivo comparar os dois grupos de idosos, sendo aplicados métodos estatísticos descritivos e inferenciais. Para os testes de hipóteses, foi pré-fixado o nível de significância $\mathrm{a}=0.05$ para rejeição da hipótese de nulidade. O teste estatístico de Wilcoxon (teste pareado) permitiu analisar as diferenças antes e depois da intervenção (AYRES et al., 2008). O teste U de Mann-Whitney (teste para amostras independentes) mediu a diferença entre as medianas entre os dois grupos (AYRES et al., 2008). Todo o processamento estatístico foi realizado sob o suporte computacional do Programa BioEstat versão 5.0. Os valores significantes foram assinalados por $(*)$.

A média de idade dos sujeitos foi de 73,83 anos. Tanto no grupo experimental quanto no grupo controle, $66,67 \%$ dos idosos tinham idade entre 60 a 80 anos e somente $33,33 \%$ com mais de 80 anos.

Em relação ao sexo dos idosos, tanto no grupo experimental quanto no controle, $66,67 \%$ eram do sexo masculino. Esses resultados são discordantes da maioria dos estudos, que apontam como característica populacional brasileira que a mulher idosa tem maior número nas ILPI (CHAIMOWICZ, 1997; CAMARANO, 2002).

O tempo médio de institucionalização era de 5,33 anos. No grupo experimental, todos idosos (100\%) apresentaram tempo de institucionalização de 1 a 5 anos. Já no grupo controle, a metade (50\%) dos idosos tinha tempo de institucionalização de 1 a 5 anos e a outra metade acima de 5 anos. Estudos de Chaimowicz (1997) e Camarano (2002) ressaltam que mulheres passam mais tempo institucionalizadas, em comparação aos homens.
Quanto aos motivos de institucionalização, foram citados dois principais: risco social e ausência de referência familiar. No grupo experimental, 66,67\% idosos apresentaram risco social, enquanto que, no grupo controle, $50 \%$. Foram incluídos no aspecto "risco social", idosos que relataram ter sofrido violência na própria casa, nas ruas ou até nos serviços de saúde oferecidos pelo governo. Para Chaimowicz (1997), Mazza e Lefevre (2004), estes são os principais motivos que levam o idoso às instituições, em todo o Brasil.

Estes achados corroboram com pesquisas nacionais na área de violência contra o idoso, que indicam que grande parte dos idosos brasileiros já sofreu com este tipo de situação, foram ofendidos, tratados com ironia ou gozação ou foram humilhados, menosprezados, ameaçados ou aterrorizados (SESC Nacional, 2007). Segundo Oliveira e Guariento (2008), grande parte dos idosos em situação de risco social apresenta não só déficit cognitivo como também funcional, freqüentemente associado à depressão.

Em relação ao recebimento de visitas, a maioria dos idosos institucionalizados $(66,67 \%)$, tanto do grupo experimental quanto do grupo controle, não recebe visitas. Esses dados estão em consonância com os estudos realizados em Dourados no Mato Grosso do Sul, e em Jequié na Bahia, por Reis, Torres e Reis (2008),nos quais apenas $20 \%$ dos idosos abrigados recebiam visitas, reforçando sentimentos de abandono e solidão, que são fatores de risco para quadros depressivos.

Quanto à naturalidade dos sujeitos, todos (100\%) idosos do grupo experimental eram oriundos do interior do Estado do Pará e, do grupo controle, $50 \%$ dos idosos. As estatísticas nacionais confirmam esses achados, apontando que a metade dos idosos asilados nasceu e passou a maior parte de suas vidas no campo - ou viveu no campo tanto quanto nas cidades (COELHO et al., 2007).

No grupo experimental, todos (100\%) idosos recebiam aposentadoria, enquanto que, no grupo controle, $83,33 \%$ dos idosos não recebiam aposentadoria. Segundo pesquisa do SESC Nacional (2007), quase toda população idosa tem alguma fonte própria de renda, sobretudo a aposentadoria.

Quanto à escolaridade, todos (100\%) idosos dos grupos experimental e controle são analfabetos. Esse aspecto está em consonância com as pesquisas realizadas na área da gerontologia, as quais descrevem que uma grande parcela desta população não possui escolaridade (MACIEL; GUERRA, 2007; REIS et al., 2008). Alguns autores acreditam que a escolaridade também influencia no desempenho cognitivo (DURANTE, 2003; STEIN, 2005). 


\section{Desempenho cognitivo}

Os resultados encontrados pelo Mini Exame do Estado Mental, utilizado antes e após a intervenção, em ambos os grupos, mostraram que o grupo experimental apresentou uma melhora significativa no desempenho cognitivo após a intervenção, com p-valor $=0,0277^{*}$, antes $($ mediana $=16)$ e depois $($ mediana $=22,5)$. Enquanto que o grupo controle não mostrou alteração significativa, $\mathrm{p}$-valor $=0,0679$, antes $($ mediana $=14,5)$ e depois $($ mediana $=12)$. Os achados estão na Tabela 1.

Tabela 1. Desempenho cognitivo dos idosos conforme o Mini Exame do Estado Mental

\begin{tabular}{lllll}
\hline & \multicolumn{2}{l}{ Grupo Experimental $(\mathbf{n}=\mathbf{6})$} & \multicolumn{2}{l}{ Grupo Controle $(\mathbf{n}=\mathbf{6})$} \\
& Antes & Depois & Antes & Depois \\
\hline Min-Máx & $6-24$ & $11-27$ & $6-19$ & $6-16$ \\
Mediana & 16 & 22.5 & 14.5 & 12 \\
P25-P75 & $10.5-17.75$ & $21-24$ & $11.5-17.5$ & $11-14.5$ \\
Média \pm dp & $14.8 \pm 6.5$ & $21.3 \pm 5.5$ & $13.8 \pm 4.9$ & $12.0 \pm 3.6$ \\
\hline Fonte: Pesquisa de campo, 2009. & & &
\end{tabular}

A comparação entre os grupos, na situação inicial do estudo, apontou que não havia diferença estatisticamente significante, $\mathrm{p}$-valor $=0,8728$. Quando se estabeleceu nova comparação entre o grupo experimental e controle, após o período de intervenção, verificou-se que o grupo experimental (mediana $=22,5)$ obteve uma melhora significativa no desempenho cognitivo, em relação ao grupo controle $($ mediana $=12)$, com $\mathrm{p}$-valor $=0,0250^{*}$.

Diversos estudos indicam que ocorrem significativas mudanças nos resultados do Mini Exame do Estado Mental, após o período de intervenção, como Bottino et al. (2002), utilizando a reabilitação cognitiva.

O Mini Mental também foi usado em estudo realizado por Stein (2005), que verificou, ao longo do tempo, uma pequena tendência de decréscimo no desempenho cognitivo geral de idosos, sendo a média, na etapa I, de 21,08 e, na etapa II, de 19,66, em concordância com o que ocorreu no grupo controle.
Nos resultados obtidos no Teste de Fluência Verbal, que também avaliou a cognição, verificou-se que, em relação ao grupo experimental, o resultado do $\mathrm{p}$-valor $=0,0431^{*}$ (muito significativo) indicou melhora no desempenho cognitivo após a intervenção, antes (mediana $=8)$ e depois (mediana $=13$ ). No grupo controle, o resultado do p-valor= 0.3452 (não significativo) aponta que não houve melhora do desempenho cognitivo, antes (mediana $=4,5$ ) e depois (mediana $=4$ ), ambos referentes ao número de animais lembrados.

A comparação entre os grupos, na situação inicial do estudo, indicou que não havia diferença estatisticamente significante, $\mathrm{p}$-valor $=0,1093$. Quando se estabeleceu nova comparação entre o grupo experimental e controle, após a intervenção, verificou-se que o grupo experimental $($ mediana $=13)$ obteve melhor desempenho cognitivo, em relação ao grupo controle $($ mediana $=4)$, com p-valor $=$ $0,0082 *$. Os dados estão na Tabela 2.

Tabela 2. Desempenho cognitivo dos idosos conforme o Teste de Fluência Verbal (animais)

\begin{tabular}{lllll}
\hline \multirow{2}{*}{$\begin{array}{l}\text { N de palavras } \\
\text { Animais }\end{array}$} & \multicolumn{2}{l}{ Grupo Experimental $(\mathbf{n}=\mathbf{6})$} & \multicolumn{2}{c}{ Grupo Controle (n=6) } \\
Antes & Depois & Antes & Depois \\
\hline Min-Máx & $5-10$ & $5-20$ & $0-15$ & $2-6$ \\
Mediana & 8 & 13 & 4.5 & 4 \\
P25-P75 & $5.75-8.75$ & $9.75-16.25$ & $3.25-5$ & $3.25-4.75$ \\
Média $\pm d p$ & $7.5 \pm 2.1$ & $12.8 \pm 5.4$ & $5.3 \pm 5.1$ & $4.0 \pm 1.4$ \\
\hline Fonte: Pesquisa de campo, 2009. & & &
\end{tabular}

Em relação às habilidades da linguagem, estudos apontam que é uma das habilidades mais resistentes à degeneração pela idade (STEIN, 2005), ratificado pelo desempenho do grupo controle, em relação ao teste de fluência 
LOUREIRO, A. P. L. et al. Reabilitação cognitiva em idosos. Rev. Ter. Ocup. Univ. São Paulo, v. 22, n. 2, p. 136-144, maio/ago. 2011.

verbal, muito sensível para avaliar o declínio cognitivo (RADANOVIC et al., 2007).

Ferrari et al. (1997) detectou, em sua pesquisa, uma real melhora na pontuação do teste de fluência verbal, após intervenção de reabilitação cognitiva. Os resultados obtidos no grupo experimental também demonstraram a validade da intervenção de reabilitação cognitiva na melhoria do teste de fluência verbal.

Os resultados encontrados pelo Teste do Relógio, utilizado antes e após a intervenção, em ambos os grupos, mostraram que, avaliando o grupo experimental, não houve significância estatística após a intervenção, com p-valor = 0.1088 . No grupo controle, ocorreu comportamento equivalente ao grupo experimental, com p-valor $=0.9998$ (não significativo).

Antes do início da intervenção, verificou-se que não havia uma diferença estatisticamente significante entre os dois grupos, com p-valor $=0.4233$. Após a intervenção, observou-se resultado semelhante, com p-valor $=0.9362$ (não significativo). Os achados encontram-se na Tabela 3.

Tabela 3. Desempenho cognitivo dos idosos conforme o Teste do Relógio

\begin{tabular}{lcccc}
\hline & \multicolumn{2}{c}{ Grupo Experimental $(\mathbf{n}=\mathbf{6})$} & \multicolumn{2}{c}{ Grupo Controle (n=6) } \\
& Antes & Depois & Antes & Depois \\
\hline Min-Máx & $0-1$ & $0-2$ & $0-2$ & $0-2$ \\
Mediana & 0 & 1 & 0.5 & 0.5 \\
P25-P75 & $0-0.75$ & $0.25-1$ & $0-1.75$ & $0-1.75$ \\
Média \pm dp & $0.3 \pm 0.5$ & $0.8 \pm 0.8$ & $0.8 \pm 1.0$ & $0.8 \pm 1.0$ \\
\hline
\end{tabular}

Fonte: Pesquisa de campo, 2009.

\section{Capacidade funcional}

Os resultados obtidos pela utilizada antes e após a intervenção, em ambos os grupos, foram separados em duas categorias: "independentes" e "dependentes". $\mathrm{Na}$ primeira categoria, verificou-se que, no grupo experimental, o resultado do $\mathrm{p}$-valor $=0.3173$ indica que não houve significância estatística na capacidade funcional dos idosos após o tratamento, antes (mediana $=1.0$ ) e depois (mediana=1.5). Porém, ressalta-se um aumento de 0.5 na mediana, apontando alteração positiva.

Já no grupo controle, da categoria "independente", o resultado do $\mathrm{p}$-valor $=0.1797$ (não significativo) também mostra que não houve significância estatística na capacidade funcional dos idosos após o tratamento, antes (mediana=2.5) e depois (mediana $=2.0$ ). Pode ser observada, ainda, quando se analisa a mediana, uma diminuição de 0.5 , indicando alteração negativa. Os dados estão na Tabela 4.

Tabela 4. Capacidade Funcional dos idosos (categoria independentes), conforme Escala Katz

\begin{tabular}{lcccc}
\hline & Grupo Experimental $(\mathbf{n}=\mathbf{6})$ & \multicolumn{2}{c}{ Grupo Controle (n=6) } \\
& Antes & Depois & Antes & Depois \\
\hline Min-Máx & $0-7$ & $0-7$ & $0-7$ & $0-7$ \\
Mediana & 1 & 1.5 & 2.5 & 2 \\
P25-P75 & $1-2.5$ & $1-2.75$ & $0.25-5.5$ & $0-4.75$ \\
Média \pm dp & $2.2 \pm 2.6$ & $2.3 \pm 2.5$ & $3.0 \pm 3.1$ & $2.7 \pm 3.1$ \\
\hline
\end{tabular}

Fonte: Pesquisa de campo, 2009.

Na segunda categoria, "dependente", no grupo experimental, o resultado do $\mathrm{p}$-valor $=0.1088$ aponta que não houve uma real melhora da capacidade funcional dos idosos após a intervenção, antes (mediana $=1.0)$ e depois (mediana $=0.0$ ). Contudo, a mediana foi alterada em 1.0, apontando alteração positiva. No grupo controle, o resultado do $\mathrm{p}$-valor $=0.3173$ (não significativo) indica que não houve melhora da capacidade funcional dos idosos após a intervenção, quando se compara antes (mediana $=0.0)$ e depois (mediana
$=0.0$ ), ou seja, não houve alterações na mediana.

A comparação entre os grupos, na situação inicial do estudo, apontou que não havia diferença estatisticamente significante, com p-valor $=0.2298$. Após a intervenção, o resultado do $\mathrm{p}$-valor $=0.8728$ indicou também que não houve uma real melhora de um grupo em relação a outro.

Essa sutil influência na execução das atividades de vida diária, na categoria independente, demonstrou o favorecimento da independência e a diminuição da 
LOUREIRO, A. P. L. et al. Reabilitação cognitiva em idosos. Rev. Ter. Ocup. Univ. São Paulo, v. 22, n. 2, p. 136-144, maio/ago. 2011.

dependência nos idosos, que foi relatada ainda no estudo realizado por Ferrari et al. (1997), que verificou esta relação, após o desenvolvimento de um grupo de terapia de memória.

Foi observado em Tóquio, no Japão, que o declínio da performance cognitiva era proporcionalmente observado em relação à capacidade funcional em 313 idosos de uma comunidade (IWASA et al., 2008).

Também outros pesquisadores, como Dirik, Cavlak e Akdag (2006), em estudo realizado em uma casa de repouso, na Turquia, verificaram que a diminuição da capacidade cognitiva influenciava negativamente o estado de independência funcional e mobilidade.

Na região nordeste do Brasil, Maciel e Guerra (2007) afirmam que o declínio cognitivo é o maior preditor de incapacidade funcional. Fiedler e Peres (2008), em pesquisas com idosos no sul do Brasil, constataram que um dos fatores associados à perda da capacidade funcional é o declínio cognitivo. Na região metropolitana de Belo Horizonte,
Giacomin et al. (2008) perceberam que o declínio cognitivo é um constante fator associado à incapacidade funcional.

Os resultados obtidos pelo Questionário de Atividades Funcionais de Pfeffer, utilizado antes e após a intervenção, em ambos os grupos, indicaram que o grupo experimental apresentou uma melhora significativa na capacidade funcional dos idosos, após a intervenção, com $\mathrm{p}$-valor $=0.0431 *$ (muito significativo), antes (mediana $=23$ ) e depois (mediana $=24,5)$. Enquanto que o grupo controle não mostrou alteração significativa na capacidade funcional, antes $($ mediana $=15)$ e depois (mediana $=14.5)$, com $\mathrm{p}$-valor $=0.7874$.

A comparação entre os grupos, na situação inicial do estudo, apontou que não havia diferença estatisticamente significante, com $p$-valor $=0.3785$. Após a intervenção, $o$ resultado do $\mathrm{p}$-valor $=0.2623$ indicou que não houve uma significativa melhora do grupo experimental em relação ao grupo controle, mesmo ocorrendo mudança na mediana. Os resultados estão na Tabela 5 .

Tabela 5. Capacidade funcional dos idosos conforme o Questionário de Atividades Funcionais de Pfeffer

\begin{tabular}{lllll}
\hline & \multicolumn{2}{l}{ Grupo Experimental $(\mathbf{n = 6})$} & \multicolumn{2}{c}{ Grupo Controle (n=6) } \\
& Antes & Depois & Antes & Depois \\
\hline Min-Máx & $3-30$ & $6-30$ & $11-19$ & $5-24$ \\
Mediana & 23 & 24.5 & 15 & 14.5 \\
P25-P75 & $8.5-27.75$ & $12.75-29.5$ & $13.25-17.5$ & $14-16.5$ \\
Média \pm dp & $18.7 \pm 12.0$ & $20.8 \pm 10.6$ & $15.2 \pm 3.1$ & $14.8 \pm 6.1$ \\
Fonte: Pesquisa de campo, 2009. & & &
\end{tabular}

Os instrumentos utilizados para avaliar a capacidade funcional, ou seja, atividades de vida diária e atividades instrumentais de vida diária, mesmo com a melhora no desempenho cognitivo dos idosos, não obtiveram resultados estatisticamente significativos. Porém, verificou-se uma diferença na mediana, indicando alteração positiva.

Esse resultado pode ser explicado pelo fato do Questionário de Pffefer, diferente da Escala de Katz, permitir uma suposta realização da atividade e, dentro do perfil de idosos institucionalizados, como nem sempre eles têm a oportunidade de realizar certas tarefas, pela própria organização institucional, o Questionário pode ser mais adequado para avaliação de idosos em instituição.

Nikolava, Demers e Béland (2009), em Montreal, no Canadá, realizaram um estudo que avaliou idosos, nos intervalos de 12 e 36 meses, utilizando medidas estatísticas repetidas, para funções cognitivas e funcionais, nos domínios das atividades de vida diária e atividades instrumentais de vida diária. Verificaram uma substancial deterioração cognitiva influenciando na perda da capacidade funcional, a longo prazo, especialmente nas atividades instrumentais de vida diária, observaram uma deterioração bem mais significativa, explicada pela complexidade dessas tarefas.

Neste estudo, provavelmente como consequência da intervenção, verificou-se um resultado mais significativo em relação às atividades instrumentais de vida diária, concordando com o estudo canadense, já que a melhora cognitiva, pode influenciar mais rapidamente nas atividades instrumentais.

\section{CONCLUSÃO}

Este estudo evidencia que o contexto asilar tem influência nos aspectos do desempenho ocupacional do idoso, principalmente no âmbito cognitivo. Os resultados 
LOUREIRO, A. P. L. et al. Reabilitação cognitiva em idosos. Rev. Ter. Ocup. Univ. São Paulo, v. 22, n. 2, p. 136-144, maio/ago. 2011.

encontrados demonstraram que houve uma evolução no desempenho cognitivo, refletindo na capacidade funcional dos idosos institucionalizados de forma sutil. A melhora do desempenho dos participantes do estudo em relação à capacidade funcional, provavelmente, não foi tão significativa, por se tratar de idosos que, em sua maioria, apresentam limitação motora e dependência instalada.

Portanto, os resultados obtidos comprovam as mudanças percebidas pelas pesquisadoras, que corroboram com uma série de estudos de autores renomados. Esta constatação fortalece a importância da intervenção de terapia ocupacional no campo da reabilitação cognitiva voltada à saúde do idoso, e a ampliação de horizontes para buscar compreender a relação entre o desempenho cognitivo e a capacidade funcional em idosos institucionalizados.

Este estudo confirma a necessidade de haver no interior das instituições asilares, o desenvolvimento de atividades intelectuais, físicas, religiosas, sociais que motivem o idoso asilado, diminuindo sua inatividade e estimulando-o ao convívio social e à busca de sentido para o seu dia-a-dia.

LOUREIRO, A. P. L., LIMA, A. A., SILVA, R. C. G, NAJJAR, E. C. A. Cognitive rehabilitation on elderly institutionalized people: a pilot study. Rev. Ter. Ocup. Univ. São Paulo, v. 22, n. 2, p. 136-144, maio/ago. 2011.

\begin{abstract}
This study describes the effects of occupational therapy on cognitive performance and its influence on the functional capabilities of older institutionalized persons. The research design was random, descriptive and longitudinal, and conducted in a long term public institution for the elderly. The data were based on results from the following measures: For cognitive abilities, a Socio-Demographic Questionnaire, the Mental State Mini-Exam, a Verbal Fluency Test, and the Clock Test. For functional capacity, the Katz Scale of Daily Living Activity and the Pfeffer Questionnaire of Functional Activities was used. Old people in the experimental group underwent 23 cognitive training sessions. The mean level of cognitive performance of the experimental group differed significantly from that of the control group; however, there was no significant betweengroup difference for emotional capacity. In conclusion, the use of occupational therapy exerted significant effects on the cognitive performance of institutionalized older persons, and although the therapy produced some positive outcomes in functional capacity, the inter-group difference did not reach statistical significance.
\end{abstract}

KEY WORDS: Rehabilitation; Cognitive therapy/methods; Aged; Frail elderly; Health management; Health of institutionalized elderly; Occupational therapy.

\title{
REFERÊNCIAS
}

AMERICAN OCCUPATIONAL THERAPY ASSOCIATION. Management of occupational therapy services for persons with cognitive impairments (statement). Am. J. Occup. Ther., v. 53 n. 6, p. 601-607, 1999.

ARRIBADA, M.C. Occupational therapy in rheumatology. Rev. Chil Reumatol.; v. 20, n.4, p. 183-183, 2004.

AYRES, M. ,et. al. BioEstat 5: aplicações estatísticas nas áreas das ciências biológicas e médicas. 5a. ed. Belém, PA: Publicações Avulsas do Mamirauá, 2008.

BRASIL. Portaria $n^{\circ} 810 / 1989$. Normas para funcionamento de casas de repouso, clínicas geriátricas e outras instituições destinadas ao atendimento do idoso. Brasília: MS, 1989.

BRASIL. Portaria SAS - 073 de 10 de maio de 2001. Normas de funcionamento de serviços de atenção ao idoso no Brasil. Brasília: MS, 2001.

CAMARA, V. D.; et al. Reabilitação cognitiva das demências. Rev. Bras. Neurol., v. 45, n. 1, p. 25-33, 2009.

CAMARANO, A. A. Envelhecimento da população brasileira: uma contribuição demográfica. Rio de Janeiro: Instituto de Pesquisa Econômica Aplicada, 2002.

CARDOSO, A. P.; FREITAS, L. C.; TIRADO, M. G. A. Oficina de som e movimento: um espaço de intervenção terapêutica ocupacional. Rev. Ter. Ocup. Univ. São Paulo, v. 13, n. 2, p. 5155,2002

CAVAlCANTI, A.; GALVÃO, C. Terapia ocupacional: fundamentação e prática. Rio de Janeiro: Guanabara Koogan, 2007. 
LOUREIRO, A. P. L. et al. Reabilitação cognitiva em idosos. Rev. Ter. Ocup. Univ. São Paulo, v. 22, n. 2, p. 136-144, maio/ago. 2011.

CHAIMOWIC, F. A saúde dos idosos brasileiros às vésperas do século XXI: problemas, projeções e alternativas. Rev. Saúde Pública, v. 31, n.2, p. 184-200, 1997.

COELHO, J. L. A.; CANTANHEDE, A. H. N.; NEGRÃO, B. C.; et al. Perfil do idoso atendido em "Casa Família Mangueirão" - SUS. Rev. Paraense Med., Belém, v. 21, n. 1, p. 48-58, 2007.

DIRIK, A.; CAVLAK, U.; AKDAG, B. Identifying the relationship among mental status, funcional independence and mobility level in Turkish institutionalized elderly: Gender differences. Arch. Gerontol. Geriatr., v. 42, p. 339-350, 2006.

DURANTE, A. M. G. Prevalência de déficit cognitivo em idosos residentes no domicílio em área urbana de Montes Claros-MG. 2003. Dissertação (Mestrado) - Escola Paulista de Medicina, UNIFESP - Universidade Federal de São Paulo, São Paulo, 2003.

FERRARI, M. A. C.; et al. Estimulação cognitiva na terceira idade/ Third age cognitive stimulation. Rev. Ter. Ocup., v. 8, n. 2/3, 1997 .

FREITAS, E. V.; PY, L.; NERI, A. L.; et al. (Org.). Tratado de geriatria e gerontologia. Rio de Janeiro: Guanabara Koogan, 2002.

GIACOMIN, K. C., et al. Estudo de base populacional dos fatores associados à incapacidade funcional entre idosos na Região Metropolitana de Belo Horizonte, Minas Gerais, Brasil. Cad. Saúde Pública, v. 24, n. 6, p. 1260-1270, 2008.

IWASA, H. et al. Cognitive performance as a predictor of funcional decline among the non-disabled elderly dewelling in a Japanese community: A 4-year population-based prospective cohort study. Arch. Gerontol. Geriatr., v. 47, p. 139-149, 2008.

HARGREAVES, L. H. H. Geriatria. Brasília: Seep, 2006.

JOÃO, A. F.; SAMPAIO, A. A. Z.; SANTIAGO, E. A.; et al. Atividades em grupo: alternativa para minimizar os efeitos do envelhecimento. Textos sobre envelhecimento, Rio de Janeiro, v. 8, n. 3, p. 1-10, 2005.

KATZ, S.; et. al. Progress in development of the index of ADL. Gerontologist,; v.10, n.1, p.20-30, 1970.

MACIEL, A. C.; GUERRA, R. O. Influência dos fatores biopsicossociais sobre a capacidade funcional de idosos residentes no nordestes do Brasil. Rev. Bras. Epidemiol., v. 10, n. 2, p. 178189, 2007.

MAZZA, M. M. P. R.; LEFEVRE, F. A instituição asilar segundo o cuidador familiar do idoso. Saúde Sociedade, v. 13, n. 3, 2004. Disponível em: availablefrom $<$ http:// www.scielo.br/scielo.php?script $=$ sci_arttext\&pid $=$ S0104$12902004000300008 \& \operatorname{lng}=\mathrm{en}_{\mathrm{N}} \mathrm{nrm}=\mathrm{iso}>$. Acesso em: 13 jul. 2009.

Recebido para publicação: 10/06/2011

Aceito para publicação: 10/07/2011
NIKOLAVA, R.; DEMERS, L.; BÉLAND, F. Trajectories of cognitive decline and functional status in the frail older adults. Arch. Gerontol. Geriatr., v. 48, n. 1, p. 28-34, 2009.

OLIVEIRA, I. M.; GUARIENTO, M. E. Caracterização do status funcional, cognitivo e das manifestações depressivas em idosos atendidos em ambulatório de serviço de referência. Anais XVI Congresso Interno de Iniciação Cientifica. Faculdades de Ciências Médicas. Universidade de Campinas. CNPQ. 2008.

OTERO, J. L.; SCHEITLER, L. F. La rehabilitación de los transtornos cognitivos. Rev. Med Uruguay, v. 17, n. 2, p. 133$139,2001$.

PFEFFER, R. I.; et al. Measurement of functional activities in older adults in the community. J. Gerontolo., v. 7, p. 323-329, 1982.

RADANOVIC, M.; et.al. Analysis of brief language tests in the detection of cognitive decline and dementia. Dementia Neuropsychol., v. 1, n. 1, p. 37-45, 2007.

REIS, L. A.; TORRES, G. V.; REIS, L. A. Características sóciodemográficas e de saúde de idosos de uma instituição do município de Jequié/BA. Rev. Espaço Saúde, Londrina, v. 9, n. 2, p. 39-46, 2008.

SANTANA, J.; et al. Envelhecimento, lazer e instituições de longa permanência. In: Anais - IX Seminário O Lazer em debate. Belo Horizonte: Imprensa Universitária da UFMG, 2008. p. 309-16.

SANTOS, N. M. W. Etapas psicológicas da vida humana e envelhecimento saudável, segundo a Weltanschauung da Psicologia Analítica. Rev. Bras. Ciênc. Envelhecimento Humano, v. 3, n. 2, p.11-21, 2006.

SERVIÇO SOCIAL DO COMERCIO NACIONAL (SESCNacional). Perfil sócio-demográfico dos idosos brasileiros. Idosos no Brasil: vivências, desafios e expectativas na 3 a idade. 2007

SOUSA, R. G.; LOVISI, G. M. Avaliação de déficits cognitivos em moradores com mais de 65 anos de um albergue público. Revista Psiquiatr. Clín. ,São Paulo, v. 34, n. 5, p. 205-209, 2007.

STEIN, L.M. Habilidades cognitivas em indivíduos muito idosos: um estudo longitudinal. Cad. Saúde Pública, v.21 n.1, p.64-72, 2005.

TIRADO, M. G.; DRUMMOND, A. F. Intervenções do terapeuta ocupacional em instituições de longa permanência para idosos. In: DRUMMOND, A. F.; REZENDE, M. B. (Org.). Intervenções em Terapia Ocupacional. Belo Horizonte: UFMG, 2008. p. 159175

VERAS, R. Em busca de uma assistência adequada à saúde do idoso: revisão da literatura e aplicação de um instrumento de detecção precoce e de previsibilidade de agravos. Cad. Saúde Pública, Rio de Janeiro, v. 19, n. 3, p. 705-715, 2003. 